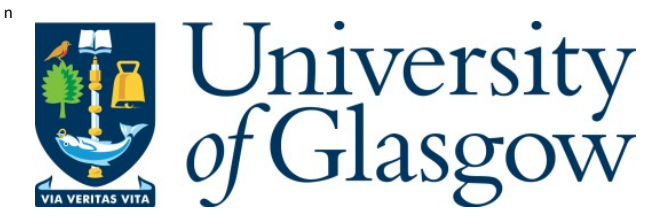

Griffiths, I.M ., K umar, A ., and Stewart, P.S. (2014) A combined network model for membrane fouling. J ournal of Colloid and Interface Science, 432 . pp. 10-18. ISSN 0021-9797

Copyright @ 2014 Elsevier

A copy can be downloaded for personal non-commercial research or study, without prior permission or charge

Content must not be changed in any way or reproduced in any format or medium without the formal permission of the copyright holder(s)

http://eprints.gla.ac.uk/94414/

Deposited on: 16 J anuary 2014

Enlighten - Research publications by members of the University of Glasgow http://eprints.gla.ac.uk 


\title{
A combined network model for membrane fouling
}

\author{
I. M. Griffiths ${ }^{\text {a,* }}$, A. Kumar ${ }^{\text {b }}$, P. S. Stewart ${ }^{\mathrm{c}}$ \\ ${ }^{a}$ Mathematical Institute, Radcliffe Square, Oxford, OX2 6GG, U.K. \\ ${ }^{b}$ Pall Corporation, Westborough, MA 01581, U.S.A. \\ ${ }^{c}$ School of Mathematics and Statistics, University of Glasgow, G12 8QW, U.K.
}

\begin{abstract}
Membrane fouling during particle filtration occurs through a variety of mechanisms, including internal pore clogging by contaminants, coverage of pore entrances, and deposition on the membrane surface. Each of these fouling mechanisms results in a decline in the observed flow rate over time, and the decrease in filtration efficiency can be characterized by a unique signature formed by plotting the volumetric flux, $\widehat{Q}$, as a function of the total volume of fluid processed, $\widehat{V}$. When membrane fouling takes place via any one of these mechanisms independently the $\widehat{Q} \widehat{V}$ signature is always convex downwards for filtration under a constant transmembrane pressure. However, in many such filtration scenarios, the fouling mechanisms are inherently coupled and the resulting signature is more difficult to interpret. For instance, blocking of a pore entrance will be exacerbated by the internal clogging of a pore, while the deposition of a layer of contaminants is more likely once the pores have been covered by particulates. As a result, the experimentally observed $\widehat{Q} \widehat{V}$ signature can vary dramatically from the canonical convex-downwards graph, revealing features that are not captured by existing continuum models. In a range of industrially relevant cases we observe a concave downwards $\widehat{Q} \widehat{V}$ signature, indicative of a fouling rate that becomes more severe with time. We derive a network model for membrane fouling that accounts for the inter-relation between fouling mechanisms and demonstrate the impact on the $\widehat{Q} \widehat{V}$ signature. Our formulation recovers the behaviour of existing models when the mechanisms are treated independently, but also elucidates the concave-downward $\widehat{Q} \widehat{V}$ signature for multiple interactive fouling mechanisms. The resulting model enables post-experiment analysis to identify the dominant fouling modality at each stage, and is able to provide insight into selecting appropriate operating regimes.
\end{abstract}

Keywords: Filtration; membrane fouling; pore clogging; caking; network model; mathematical modelling

\section{Introduction}

Understanding membrane fouling is a key goal in separation science, and is an area in which detailed mathematical modelling can provide key insight for membrane design optimization. Membrane fouling is a complex process due to interaction between membrane properties, solution composition, and process conditions [1]. Historically, in a typical filtration set-up there are four key membrane fouling mechanisms:

i. Standard blocking - small particles pass into the membrane pores and a finite number adhere to the walls causing pore constriction.

ii. Intermediate blocking - larger particles land on the membrane surface and partially cover a pore.

iii. Complete blocking - larger particles land on the membrane surface and cover a pore entirely.

\footnotetext{
*Corresponding author

Email address: ian.griffiths@maths.ox.ac.uk (I. M. Griffiths)
}

iv. Caking - a layer of particles builds up on the membrane surface, which provides a resistance in the form of an additional porous medium through which the feed must also permeate [2].

There are various ways in which fouling behaviour may be characterized. One approach is direct monitoring of foulant deposition on the surface of a membrane, which may be achieved through a variety of techniques, including microscopy, laser sensoring, reflectometry, spectroscopy, and $3 \mathrm{D}$ imaging [3-12]. A comprehensive review of various experimental techniques, capabilities and sensitivity specific to membrane protein fouling is provided in [13]. Other more recent approaches include computational fluid mechanics [14], genetic programming (GP) models [15], artificial neural network (ANN) tools for modelling highly complex and nonlinear membrane systems [16], diffusionlimited-aggregation simulation techniques [17], and multiscale modelling of protein fouling in ultrafiltration [18].

While direct measurement is a useful tool in understanding membrane fouling, a more common and widely accepted approach involves indirect measurement. For example, examining the temporal variations in volumetric flux, defined as volume per unit membrane area per unit 
time, defined here as $\widehat{Q}$, provides key insight. When filtering at constant transmembrane pressure the flux through the membrane will reduce with time as a result of fouling (Fig. 1a). A second indirect measurement that is often made is the total throughput, defined as the volume processed per unit membrane area, $\widehat{V}$, given by

$$
\widehat{V}(\hat{t})=\int_{0}^{\hat{t}} \widehat{Q}(s) \mathrm{d} s,
$$

where $\hat{t}$ denotes time. As a result of fouling we generally find that $\widehat{V}$ approaches a constant value as $\hat{t} \rightarrow \infty$ when a constant transmembrane pressure is applied (Fig. 1b). There are several articles confirming that the mode of fouling changes during the course of membrane plugging (see, for example, $[19,20])$ with the initial stages dominated by pore blocking, followed by complete blocking, while in later stages cake formation is predominantly responsible for the continued reduction in flux.

Mathematical modelling of membrane fouling dates back to 1935 [21], focusing initially on models for individual fouling mechanisms. It emerges that these mechanisms can be expressed by

$$
\frac{\mathrm{d}^{2} \hat{t}}{\mathrm{~d} \hat{V}^{2}}=c\left(\frac{\mathrm{d} \hat{t}}{\mathrm{~d} \hat{V}}\right)^{n}
$$

where $c$ is the generalized filtration constant and $n$ may take the values $2,3 / 2,1$ or 0 , which correspond respectively to complete blocking, standard pore blocking, intermediate pore blocking, and cake filtration. This model effectively describes distinct fouling mechanisms in a definite period of filtration [22-25]. However, Iritani et al. [26] reported that these individual models were unable to fit their experimental observations and indicated that a combination of effects would probably lead to better results.

Two-stage models that combine two individual fouling mechanisms sequentially are able to improve on the agreement with experiment. For example, a combination of pore blocking followed by cake-layer build-up was shown to describe the fouling of track-etched membranes by BSA [27] and proteins [28]. This approach can be generalized to other combinations of two fouling mechanisms [29] and has been further extended to capture three sequential fouling mechanisms, such as pore constriction followed by pore blocking and finally transitioning to caking [30].

In all of these cases, the indirect measurement of flux and throughput with time provide information on the overall fouling process. However, they are unable to reveal information on the dominant active fouling mechanism at any given time. Combining both of these visualizations in a graph of $\widehat{Q}$ versus $\widehat{V}$ provides a much richer understanding of the system behaviour, from which the resulting $\widehat{Q} \widehat{V}$ signature allows the dominant mode of fouling at any instant to be inferred.

Generally, when the feed is such that fouling takes place via an individual blocking mechanism such as complete blocking or caking, the $\widehat{Q} \widehat{V}$ signature is convex downwards, that is, $\widehat{Q}^{\prime}(\widehat{V})<0$ and $\widehat{Q}^{\prime \prime}(\widehat{V})>0$, where primes denote differentiation (see solid curve in Fig. 2a). In this case, the graph depicts a membrane fouling modality where the rate of flux decline reduces as more fluid volume is processed. For example, the reduction of flux of fluid due to fouling reduces the rate of arrival of foulants (or particles), and hence reduces the subsequent rate of fouling.

However, recent experiments have revealed the possibility of an alternative, concave downwards $\widehat{Q} \widehat{V}$ signature, i.e., $\widehat{Q}^{\prime}(\widehat{V})<0$ and $\widehat{Q}^{\prime \prime}(\widehat{V})<0$, as depicted by the dashed curve in Fig. 2(a). Such a graph may be identified with a regime in which the rate of fouling increases with time, in contrast to the more conventional observations. One scenario that may give rise to such a fouling behaviour is where contaminants are able to adsorb and desorb to the internal pore structure in such a way that the reduction in pore radius leads to an exacerbated flux decline (see, for example, Fig. $2 \mathrm{~b}$ and $[31,32]$ ). However, experiments suggest that such a fouling behaviour may also be observed in the absence of any such adsorption mechanisms (see, for example, [19, 29, 30, 33]). Furthermore, it is clear that such fouling behaviour is not constrained to the filtration of specific contaminants by a specific membrane, since significantly changing both the membrane type and contaminants within the feed still reveals qualitatively similar results (Fig. 2c,d).

In these cases, it is the transition between multiple fouling mechanisms during the filtration process that is thought to be responsible for the observed flux decline. Since current theories assume either that each fouling mechanism operates at a uniform rate, simultaneously and independently of the others, or that fouling mechanisms are sequential in operation, these are unable to reproduce the concave downward shapes observed experimentally through the effects of standard, intermediate, complete blocking, and caking alone. To capture such behaviour and ultimately replicate the experimentally observed graphs requires a mathematical model that caters for the inter-relation between each fouling mechanism.

In this paper we develop for the first time the foundation of a discrete network model that allows multiple inter-related blocking mechanisms to be active at any given time, including fouling caused by particulate adsorption as well as size-based pore-plugging events. Specifically, the model caters for the interplay between membrane fouling effects, which may change with system state and hence time. For instance, particle adhesion within pores may lead to a reduction in pore size that eventually enables complete pore blocking. The fouling behaviour may be characterized through a series of parameters and we explore in $\S 3$ the specific effect that each of these has on the resulting $\widehat{Q} \widehat{V}$ signature. We explore the required model ingredients that lead to the physical observations exhibited in Fig. 2. The results are used in $\S 4$ to interpret how the dominant fouling mechanism varies during a given experiment, and enables us to determine the regimes in which 
(a)
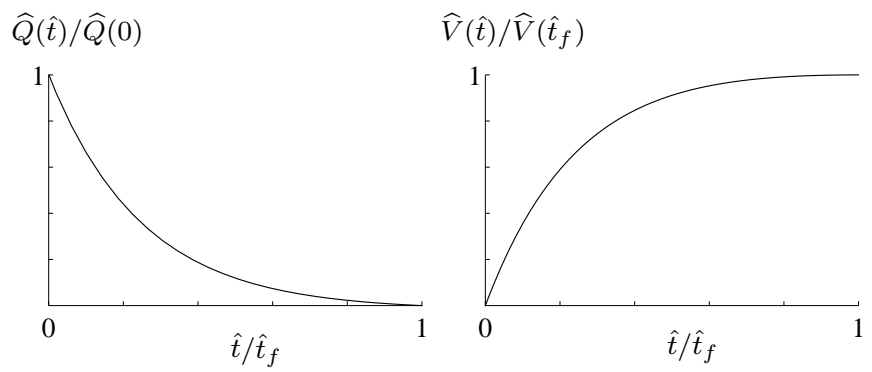

Figure 1: (a) Schematic diagram of normalized flux, $\widehat{Q}(\hat{t}) / \widehat{Q}(0)$, versus normalized time, $\hat{t} / \hat{t}_{f}$, where $\hat{t}_{f}$ is the time at which $\widehat{Q}=0$, illustrating the flux decline with time. (b) Normalized throughput, $\widehat{V}(\hat{t}) / \widehat{V}\left(\hat{t}_{f}\right)$, versus normalized time, $\hat{t} / \hat{t}_{f}$, for the same data as presented in (a).

continuum models appropriately describe the fouling behaviour, and those in which our new discrete modelling framework is essential. We conclude by generalizing our model to include more complex effects such as the ability to partially cover a pore or to land on the membrane material, and we demonstrate how this may be simply built onto our framework and the implications such features have on the physical observations.

\section{Mathematical modelling}

We consider a membrane, of uniform initial thickness $\hat{h}$, that comprises a two-dimensional $m \times n$ array of regularly spaced uniform pores each of radii $\hat{r}_{0}$, as illustrated in Fig. 3. The theory we present in this paper may easily be generalized to irregular membranes however here we choose to model a spatially homogeneous membrane to elucidate an accurate characterization of the fouling process. Such regularity is exhibited, for example, in track-etched membranes [34].

The flow rate through pore $(i, j)$ at time $\hat{t}, \hat{q}_{i, j}(\hat{t})$ is given by Poiseuille's law [35],

$$
\hat{q}_{i, j}(\hat{t})=\frac{\pi \Delta p \hat{r}_{i, j}^{4}}{8 \mu \hat{h}_{i, j}},
$$

where $\hat{r}_{i, j}(\hat{t})$ and $\hat{h}_{i, j}(\hat{t})$ are the pore radius and membrane depth respectively, which may change with time $\hat{t}$ due to the fouling mechanisms, but we assume that the fluid viscosity $\mu$ remains constant. As noted in the introduction, we consider the operating regime in which the applied pressure difference across the membrane, $\Delta p$, is constant across each pore. The total volumetric flow rate through the entire membrane area, $\widehat{Q}$, is then given by the (a)

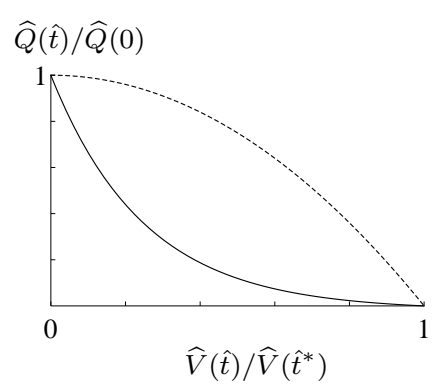

(c)

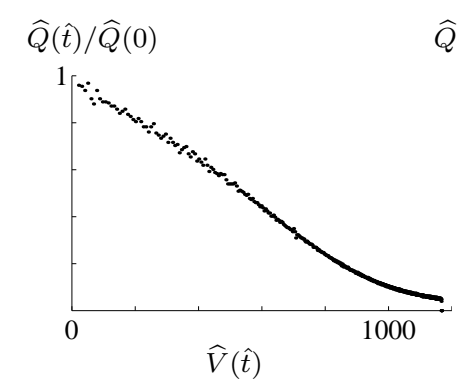

(b)

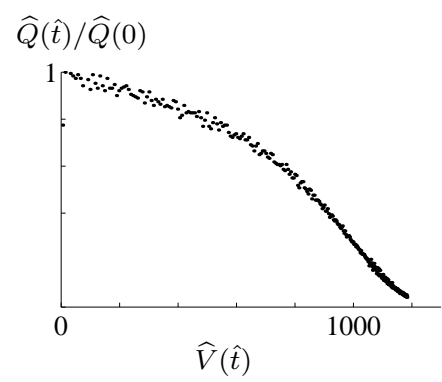

(d)

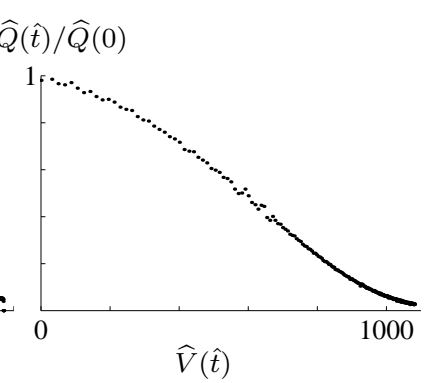

Figure 2: (a) Schematic diagram of a convex-downward (solid) and concave-downward (dashed) graph of normalized flux, $\widehat{Q}(\hat{t}) / \widehat{Q}(0)$, versus normalized throughput, $\widehat{V}(\hat{t}) / \widehat{V}\left(\hat{t}_{f}\right)$, where $\hat{t}_{f}$ is the time at which $\widehat{Q}=0$. (b) Experimental data for normalized volumetric flux versus throughput showing membrane fouling behaviour of $4 \%$ bovine serum albumin (BSA) solution filtered through a Fluorodyne $\mathbb{R}$ EX EDF Pall membrane at a constant pressure difference of 10psi. A concave-downward graph arises due to a change in fouling behaviour. (c) $2 \mathrm{~g} / \mathrm{L}$ BSA solution filtered through a $0.2 \mu \mathrm{m}$ track-etched membrane at a constant pressure difference of 20 psi. (d) $2 \mathrm{~g} / \mathrm{L} \mathrm{IgG}$ solution in PBS filtered through a $0.2 \mu \mathrm{m}$ rated sterile membrane at a constant pressure difference of 10psi. 


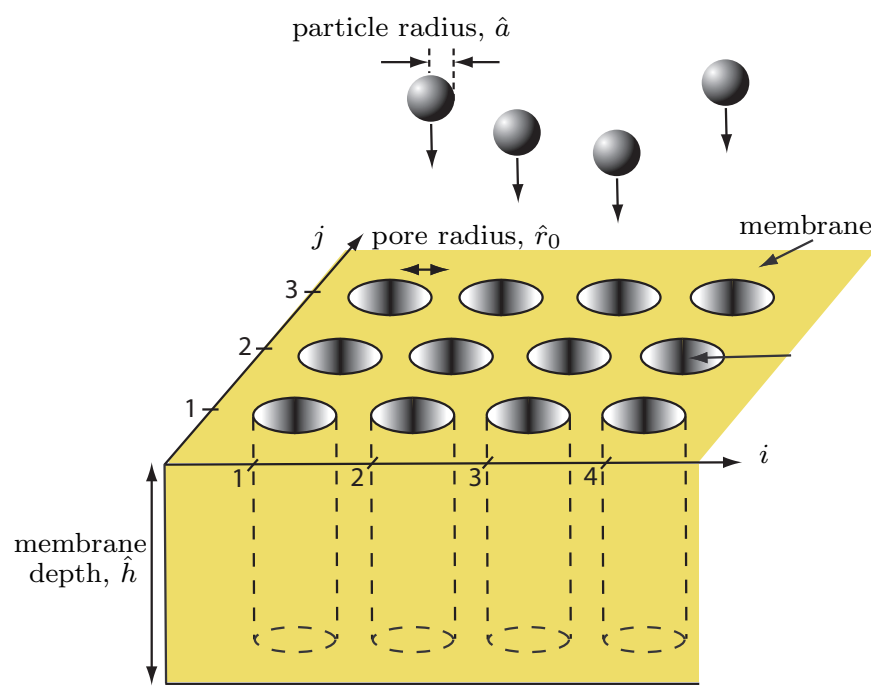

Figure 3: Schematic diagram of a portion of the membrane set-up considered. The membrane comprises a total of $m \times n$ pores.

sum of the individual fluxes through all pores,

$$
\widehat{Q}=\sum_{i=1}^{m} \sum_{j=1}^{n} \hat{q}_{i, j},
$$

and the total throughput is given by

$$
\widehat{V}=\int_{0}^{\hat{t}} \widehat{Q}(s) \mathrm{d} s .
$$

The contaminants are assumed to be solid spherical particles of constant radius, $\hat{a}$. On average, a particle will arrive at the membrane every $1 / \widehat{C} \widehat{Q}$ seconds, where $\widehat{C}$ is the concentration (number of particles per unit volume). For any given experiment the particles will, in practice, arrive at times that are randomly distributed around this expected arrival value. However, we are concerned with the general fouling that will be observed and so consider the result of the average of many numerical simulations. In doing so, any randomness in the particle arrival time that is observed for a single simulation will be smoothed out, and so it is sufficient for us to assume that particles arrive in a uniform manner with the fluid processed. All of the results presented in this paper are the average of 50 independent simulations; the results yielded were found to change very little by increasing the number of independent simulations beyond approximately 10 .

To enable comparison between different model experiments, we scale the time and flux via

$$
\hat{t}=\frac{t}{\widehat{C} \widehat{Q}(0)} \quad \hat{q}=\hat{q}(0) q(t), \quad \widehat{Q}(\hat{t})=\widehat{Q}(0) Q(t),
$$

so that, when $Q=1$, a particle will arrive on average at the membrane every unit dimensionless time. The dimensionless throughput is then

$$
V=\int_{0}^{t} Q(s) \mathrm{d} s .
$$

We also scale all lengths with the initial pore radius,

$$
\hat{r}_{i, j}=\hat{r}_{0} r_{i, j}, \quad \hat{h}_{i, j}=\hat{r}_{0} h_{i, j}, \quad \hat{h}=\hat{r}_{0} h, \quad \hat{a}=\hat{r}_{0} a .
$$

As discussed above, the flux of fluid through each pore, and thus the fouling rate, is affected by various mechanisms. In the following section we detail the effect of each of these fouling mechanisms and how they are accounted for in the network model.

\section{Routes to membrane fouling}

\subsection{Standard blocking}

If a particle of size $a$ arrives at pore $(i, j)$ and $a<r_{i, j}$ then the particle enters into the internal pore structure. Once within the pore we allow for a finite probability of particle adhesion to the pore wall, $p_{a}$. This probability will in practice be a complex function that depends on many features, such as membrane and contaminant composition and geometry. Here we suppose that the probability of adhesion within the pore, $p_{a}$ obeys the following law:

$$
p_{a}=p_{a 0}\left(1-\mathrm{e}^{-A / k}\right),
$$

where $p_{a 0}$ is a constant, $A=2 \pi r h$ is the internal surface area available onto which particles may adhere, and $k$ is a parameter that measures the importance of the available surface area: when $k=0$ the probability of adhesion is independent of available surface area. This functional form captures the key dependence on available surface area that we might expect. The probability of adhesion increases linearly with available surface area for small surface areas, but saturates at a maximum adhesion probability, $p_{a 0}$, as the available area becomes large.

If the particle is adsorbed at the wall then the constriction effect of this particle on the pore is assumed to be distributed over the entire available surface area of the pore, as illustrated in Fig. 4(a), so that the new pore radius is $\sqrt{r_{i, j}^{2}-4 a^{3} / 3 h}$. Thus, the total number of particles that may be admitted by a pore, $N^{*}$, is given by

$$
N^{*}(a, h)=\text { Ceiling }\left(\frac{3\left(1-a^{2}\right) h}{4 a^{3}}\right),
$$

where Ceiling $(x)$ denotes the ceiling function that returns the smallest integer not less than $x$. This mechanism of reduction in flux is termed standard blocking.

\subsection{Complete blocking}

If the particle of size $a$ lands on an open pore $(i, j)$ of size $r_{i, j}$ and $a>r_{i, j}$ then complete pore blocking occurs. In this case a flow, $Q^{*}$, may still continue to pass through the pore, which we identify with an imperfect seal between the particle and pore (Fig. 4b). In this paper we assume $Q^{*}$ is a constant for simplicity. 


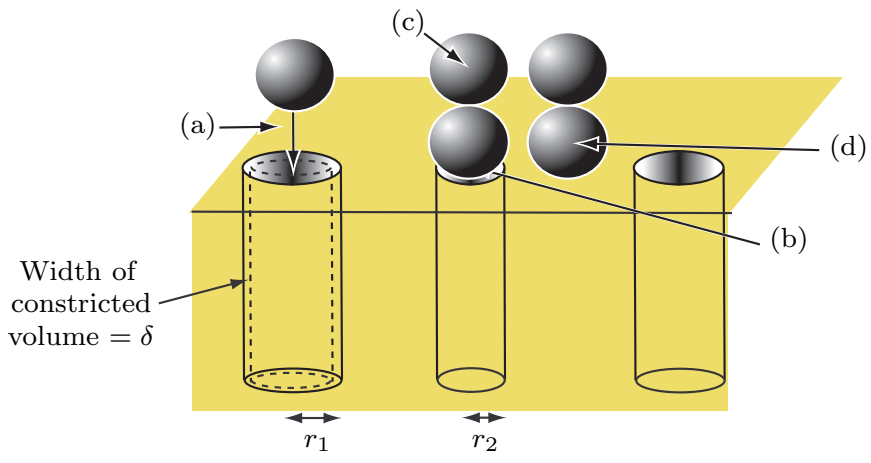

Figure 4: Schematic diagram of possible blocking mechanisms: (a) Standard blocking: Here particle volume, $4 \pi a^{3}=$ volume of constriction, $4 \pi a \delta h$ so that the resulting new pore radius = $\sqrt{r_{1}^{2}-4 a^{3} / 3 h}$. (b) Complete blocking: Here the pore radius $r_{2}<a$. (c) Caking: Here a particle lands on another particle covering a pore. (d) Landing on interpore membrane material.

\subsection{Cake filtration}

If the arriving particle of size $a$ lands on a pore that has already been blocked then this also reduces the flux through that part of the membrane by increasing the membrane thickness in Eq. (3) to $h_{i, j}+2 a$. This feature is termed caking, and is responsible for the continued reduction in flux when all pores have been blocked as illustrated in Fig. 4(c). The effect of this mechanism will be most prominent when the flux through the pore following complete pore blocking, $Q^{*}$, is comparable with the initial flux through the pore.

\subsection{Landing on interpore membrane material}

If the particle lands on interpore surface then it has no effect on the total flux but adds to the depth of the membrane layer at that position. When the fraction of particles that land on interpore material increases, the time taken for the membrane to foul will increase (Fig. 4d).

\subsection{Mathematical model for routes to membrane fouling}

Each of the fouling effects outlined above are catered for in the network model by allowing for the particle either to land on a pore or to land on membrane material. The pore selected by the particle is also determined via a weighted probability that favours sites through which there is a greater flux of fluid. Specifically, we assume that the probability of landing on a specific pore $(i, j)$, $p_{(i, j)}=p_{0} Q_{i, j} / Q$, where $p_{0}$ is a constant. The probability of landing on the membrane material is then $p_{m}=1-p_{0}$.

In all of the simulations conducted here we consider an $n=m=12$ array of pores, which was found to be sufficiently large to provide an accurate representation of an infinitely large array of pores.

\section{Results}

We first present results for a regime in which all four fouling mechanisms introduced in $\S 3$ are possible. In this

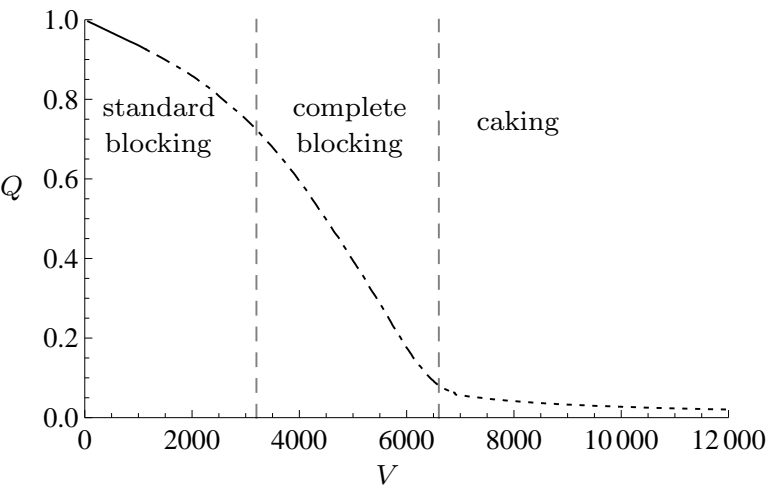

Figure 5: Flux, $Q$, versus throughput, $V$, for a feed of particles smaller than the pores, $a=0.9$, probability of adhesion, $p_{a 0}=$ 0.1 , areal-dependent adhesion parameter, $k=0$, membrane thickness $h=10$, fraction of pore leakage following complete blocking, $Q^{*}=0.3$, and probability of arriving at a pore $p_{0}=1$. Distinct regions are observed where first standard blocking is the dominant mechanism of fouling, followed by complete blocking, and finally a tail region in which the membrane continues to foul via caking. The solid line depicts the region in which no pores are fully blocked, the dot-dashed segment of the curve delineates the region where some pores are blocked, and the dotted portion of the curve shows the portion where all pores are fully blocked and fouling takes place via caking. The resulting graph exhibits both convex and concave downward regions.

case we require the feed to be composed of contaminants with radius $a<1$, so that their size is smaller than that of the membrane pores and the particles may enter the pores. We also set $Q^{*} \neq 0$ so that the effect of caking is present. We make two observations in this case. Firstly, the $Q V$ signature is able to distinguish each dominant active blocking mechanism via the concavity or convexity (Fig. 5). Specifically, we find that the graph is composed of three distinct regions: an early-time region in which the flux decline is dictated by pore constriction; a second region in which the flux falls more rapidly as a result of complete pore blocking; and finally a gradual asymptote of the flux to zero through caking. Crucially, as a result of the ability of the model to cater for the coupling among the multiple poreclogging mechanisms, this mathematical model is able to replicate the rapid flux decline observed experimentally in Fig. 2(b-d) through the concave-downward $Q V$ signature. As discussed earlier, such a trend is not captured by current continuum models that describe and combine the effects of standard blocking, complete blocking and caking in an independent way.

In the following subsections we explore the relation between the individual parameters present in our model and the resulting $Q V$ signature, and the underlying physical explanation for the behaviour observed. 
(a)

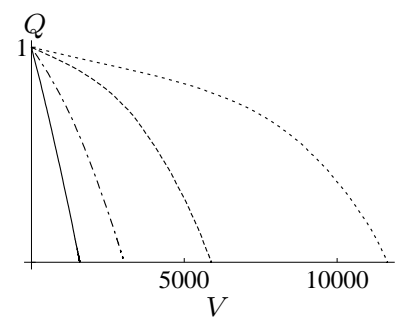

(c)

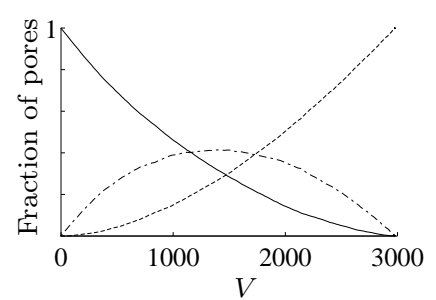

(b)

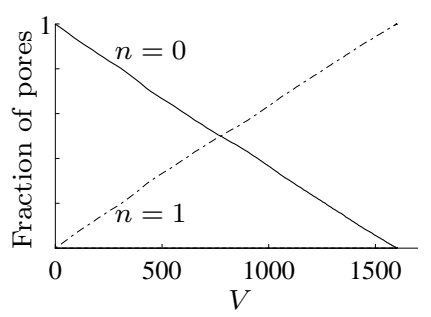

(d)

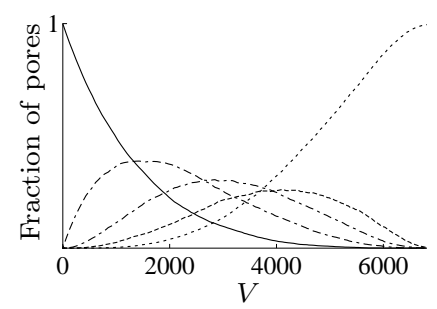

Figure 6: (a) Flux, $Q$, versus throughput, $V$, as the probability of adhesion within the internal membrane structure is increased, $p_{a 0}=0.05$ (solid), 0.1 (dot-dashed), 0.2 (dashed), 0.3 (dotted). Here we consider a feed of smaller particles than the pores, $a=0.9$, with membrane thickness $h=10, Q^{*}=0$ so there are no caking effects, probability of arriving at a pore $p_{0}=1$, and areal-dependent adhesion parameter, $k=0$. (b) Collapse of these graphs by rescaling the $x$-axis with final throughput, $V_{f}$.

\subsection{No pore leakage, $Q^{*}=0$}

An increase in the probability of adhesion of particles admitted to the pore, $p_{a 0}$, generates a more rapid flux decline and final throughput as we would expect (Fig. 6a). However, we also find that the $Q-V$ graphs exhibit selfsimilarity and we are able to collapse all graphs onto a master curve by rescaling the $x$-axis with the final throughput, $V_{f}$ (Fig. 6b). Furthermore, the total throughput is related to the probability of adhesion via

$$
V_{f} \propto p_{a 0}^{-\alpha}
$$

where $\alpha \approx 0.95$ and is independent of the particle size and membrane depth, with data lying within $0.5 \%$ of this fit for the simulations conducted.

As we increase the relative depth of the membrane compared with the pore radius the number of particles that may be accepted by each pore varies with membrane depth, $h$ in a discrete manner according to (10). As a result, we find that the $Q-V$ graph similarly varies discretely, with the time taken before complete clogging occurs increasing with increasing $h$ (Fig. 7a). The increase in the number of particles that may be accepted by a pore with increasing $h$ is apparent by examining the distribution of particles contained within each pore, which illustrates the pore-filling process with time (Fig. $7 \mathrm{~b}-\mathrm{d}$ ).

The $Q V$ signatures are again self-similar and may be collapsed to a master curve by plotting versus the throughput scaled with its final value determined from

$$
V_{f} \propto m n\left(N^{*}(a, h)+1\right)
$$

where we recall that $m n$ is the total number of pores in the membrane and $N^{*}$ is the total number of particles that may be adsorbed within a pore before the next

Figure 7: (a) Flux, $Q$, versus throughput, $V$, as the membrane depth is increased, $h=2$ (solid), 6 (dot-dashed), 20 (dashed) and 40 (dotted), so that $N^{*}=1,2,4,8$ particles respectively may be admitted by each pore before complete blocking takes place. Here we consider a feed of smaller particles than the pores, $a=0.9$, probability of adhesion, $p_{a 0}=0.1$, areal-dependent adhesion parameter, $k=0$, and $Q^{*}=0$ so there are no caking effects, probability of arriving at a pore $p_{0}=1$, and areal-dependent adhesion parameter, $k=0$. (b) Fraction of pores containing no particles (solid curve) and 1 particle (dotdashed curve) when membrane depth $h=2$ so $N^{*}=1$, illustrating the accumulation of adhered particles in each pore with volumetric flux. (c) Fraction of pores containing zero (solid), 1 (dot-dashed), and 2 (dashed) particles when $h=6$ so $N^{*}=2$. (d) Fraction of pores containing no (solid), 1 (dot-dashed-dashed), 2 (dot-dashed) 3 (dashed) and 4 (dotted) particles when $h=20$ and $N^{*}=3$.

particle completely blocks the pore, given by (10). Thus $m n\left(N^{*}(a, h)+1\right)$ denotes the total number of particles that may be accepted by a pore, either within the pore itself or on the surface, before the membrane is completely blocked. Varying the value of $k$ also reveals self-similarity, though the final throughput depends on $k$ in a nonlinear fashion, as observed in Fig. 8.

\subsection{Pore leakage, $Q^{*}>0$}

When we allow a non-zero flux, $Q^{*}$, to pass through a completely blocked pore a tail region in the $Q-V$ graph emerges, which becomes more pronounced as $Q^{*}$ increases (Fig. 9). The data can still be collapsed for the regime in which complete blocking dominates, by rescaling the throughput with the value attained when the flux reaches $Q^{*}$ (Fig. 10). However, the behaviour in the latter stages is dominated by caking and does not collapse onto a master curve.

When $Q^{*}>0$ the flux declines algebraically with throughput and thus no longer attains zero flux in finite time. We may, however, still compare the typical time to blocking by considering, for example, the throughput at which the flux has fallen to a specific (small) value. Considering the throughput attained when the flux falls 


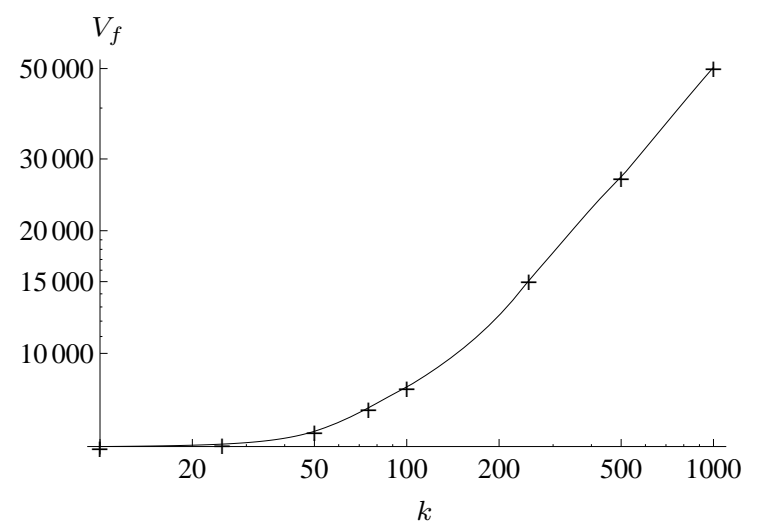

Figure 8: Total throughput, $V_{f}$, versus areal-dependent adhesion parameter, $k$, for a feed of particles smaller than the pores, $a=0.9$, probability of adhesion $p_{a 0}=0.1$, membrane thickness $h=10$, fraction of pore leakage following blocking, $Q^{*}=0$, and probability of arriving at a pore, $p_{0}=1$.

$$
Q
$$

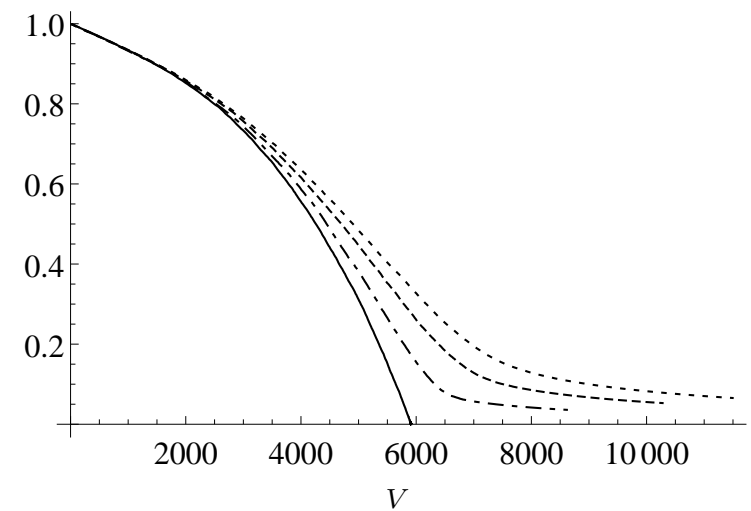

Figure 9: Flux versus throughput when $Q^{*}=0$ (solid), 0.1 (dotdashed), 0.2 (dashed) and 0.3 (dotted). Here we consider a feed of smaller particles than the pores, $a=0.9$, probability of adhesion, $p_{0}=0.1$, areal-dependent adhesion parameter, $k=0$, membrane thickness $h=10$, and probability of arriving at a pore $p_{0}=1$.

to $0.5 \%$ of its original value, $V_{0.5 \%}$, we find that this exhibits a linear dependence on $Q^{*}$ with numerical data lying within a $1 \%$ relative error of a linear fit.

\subsection{Larger particles}

When the particle radius exceeds that of the pore radius, that is, when $a>1$, standard blocking can no longer take place and the fouling occurs only through complete blocking and caking. As a result, we find that, in this regime, the curvature of the graph changes from concave to the more familiar convex structure (Fig. 11). In the case when the particles perfectly seal a pore upon arrival $\left(Q^{*}=0\right)$ the $Q-V$ graph is linear (Fig. 11). (a)

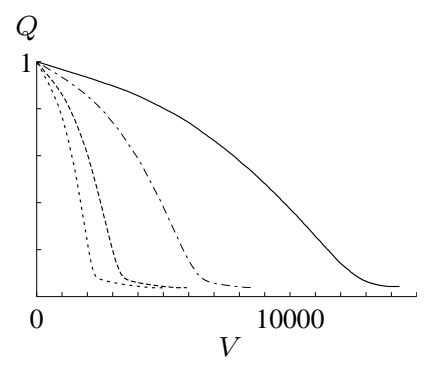

(b)

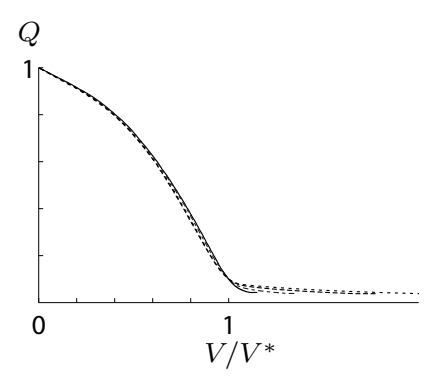

Figure 10: (a) Flux, $Q$, versus throughput, $V$, as the probability of adhesion within the internal membrane structure is increased, $p_{a 0}=0.05$ (solid), 0.1 (dot-dashed), 0.2 (dashed), 0.3 (dotted). Here we consider a feed of smaller particles than the pores, $a=0.9$, with membrane thickness $h=10$, and $Q^{*}=0.1$ so that caking is also present, probability of arriving at a pore $p_{0}=1$, and areal-dependent adhesion parameter, $k=0$. (b) Collapse of these graphs by rescaling the $x$-axis with the throughput $V^{*}$ achieved when $Q=Q^{*}=0.1$.

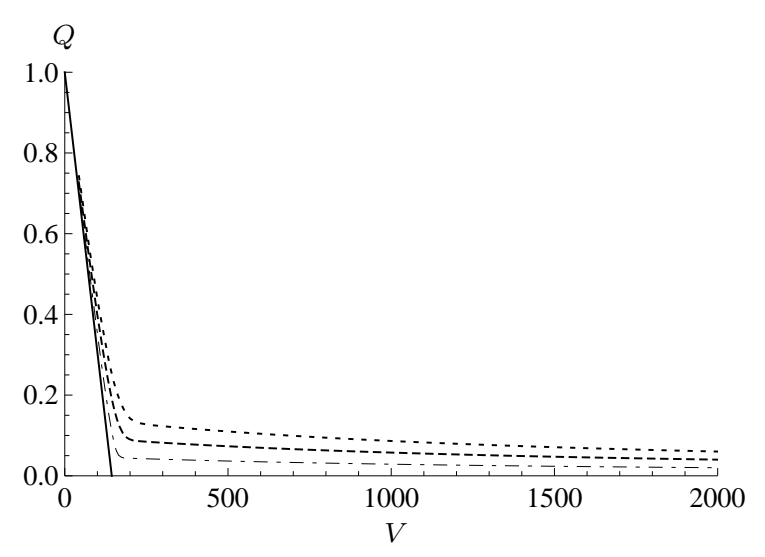

Figure 11: Flux, $Q$, versus throughput, $V$, as the permitted relative flux through a blocked pore is increased, $Q^{*}=0$ (solid), 0.01 (dotdashed), 0.05 (dashed), 0.1 (dotted). Here we consider a feed of larger particles than the pores, $a=1.1$, with membrane thickness $h=10$, and probability of arriving at a pore, $p_{0}=1$. 
(a)

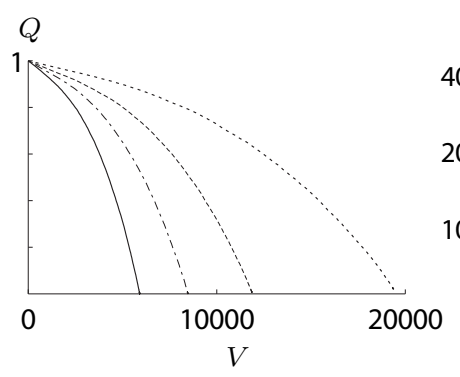

(b)

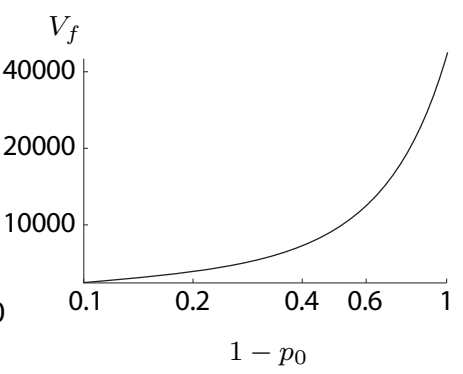

Figure 12: (a) Flux, $Q$, versus throughput, $V$, as the probability of landing on membrane material is increased, $p_{m}=1-p_{0}=0$ (solid), 0.3 (dot-dashed), 0.5 (dashed), 0.7 (dotted). (b) Final throughput, $V_{f}$, versus probability of landing on the membrane material, $1-p_{0}$. In both cases, we consider a feed of smaller particles than the pores, $a=0.9$, probability of adhesion, $p_{a 0}=0.1$, areal-dependent adhesion parameter, $k=0$, membrane thickness $h=10$, and $Q^{*}=0$ so there are no caking effects.

\subsection{Landing on the membrane material}

As discussed in $§ 3.4$, particles that land on the interpore membrane space will have no effect on the flow rate. As a result, when we allow particles to land on the membrane material, the only effect is to increase the total throughput before the membrane becomes completely blocked, so that the $Q V$ signature is stretched along the $x$ axis with no influence on the shape of the curve (Fig. 12a). This allows us again to collapse all graphs onto a single master curve by scaling appropriately, in an identical manner to that demonstrated in Fig. 12(a). In this case, the final throughput, $V_{f}$, has an approximately linear dependence on the probability of landing on the membrane material for suitably low probabilities $p_{m}=1-p_{0} \lesssim 0.4$, and diverges to infinity as $p_{m} \rightarrow 1$ (Fig. 12b). The majority of industrial microfiltration membranes have surface porosities in excess of $50 \%$. In such situations we expect the behaviour to be approximated by the linear region of the graph. For track-etched membranes the typical porosity can be controlled and may vary significantly. (For the data shown in Fig. 2c a $0.2 \mu \mathrm{m}$ track-etched membrane with surface porosity of $13 \%$ was used.)

\subsection{Partially covering a pore}

In this final section we develop our model to account for the possibility of the arriving particle also landing on the edge of an open pore and partially blocking the pore, that is, intermediate blocking. In this case the flux will be reduced, depending on the particle size. To cater for this feature mathematically we allow particles that arrive at a pore either to land in the centre, where they will enter the pore if $r>a$ or block the pore completely if $r<a$, or to land on the edge of the pore and occupy one of four sites around the edge of the membrane. We suppose that the latter event has an associated probability $p_{p}$ of occurrence. For each particle that lands on an intermediate-blocking site we suppose that the flux is reduced by a proportion $\phi<1 / 4$, so that the flux when all four sites are occupied is $(1-4 \phi)$ times that of a completely open pore. Partial coverage also brings one of two other associated physical implications:

(i). The reduction in flux does not affect the effective pore radius beneath. In this case, the particle that partially blocks the pore reduces the flux but other particles are still able to 'squeeze past' the obstruction at the entrance and adsorb onto the pore walls in the membrane.

(ii). The reduction in flux also corresponds to a reduction in the effective pore radius, which is determined via Poiseuille's law (3) for the new flux. In this case the particle that partially blocks the pore also reduces the size of subsequent particles that can be transmitted into the pore.

We expect that Rule (i) may be appropriate when the particles in the feed are sufficiently small to pass by the obstruction at the pore entrance and subsequently be transmitted into the pore, while Rule (ii) provides the most appropriate representation when the particles are comparable in size to the pore, so that they create a significant obstruction at the entrance.

If we vary the probability of partially covering a pore and suppose that Rule (i) is obeyed then we find that the $Q V$ signature of the graph is able to change from concave downwards (solid line in Fig. 2a) to the more conventional convex-downwards (dashed line in Fig. 2a) as we increase the probability of a particle partially covering a pore, $p_{p}$ (Fig. 13a). This indicates that we may be able to use the shape of the $Q V$ signature to decide whether particles will predominantly be admitted by the pore or land close to a pore and provide partial coverage. In response to the change in curvature in the $Q V$ signature, the total throughput, $V_{f}$, is observed first to increase with increasing probability of partially landing on the pore, $p_{p}$, before decreasing thereafter (Fig. 13b). As a result, we observe the existence of a maximum throughput, in this example when $p_{p} \approx 0.1$, that may be achieved for a specific probability of intermediate pore blocking. This suggests that by treating the membrane to adjust the affinity of particles with the surface we may be able to improve the total throughput achieved.

When intermediate-blocking Rule (ii) is obeyed we similarly observe the capability for the $Q V$ signature to switch from concave to convex (Fig. 14a), and again observe a non-monotonic dependence of the final throughput on the probability of partially landing on a pore. However, in this case we find that a minimum throughput is attained, for a critical probability of $p_{p} \approx 0.55$ (Fig. 14b). This suggests that in filtration scenarios where partial pore coverage reduces both the flux through the pore and the effective pore radius available for adsorption it is important to consider 
(a)

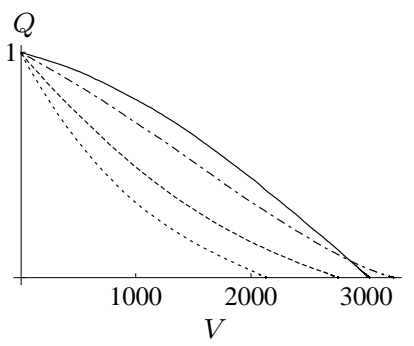

(b)

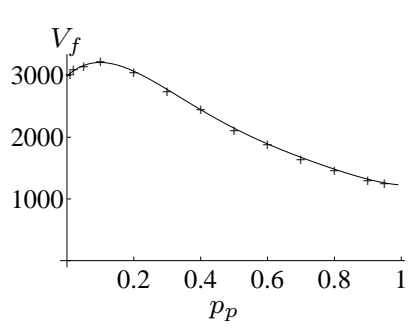

Figure 13: (a) Flux, $Q$, versus throughput, $V$, as the probability of landing partially on a pore, $p_{p}=0.1$ (solid), 0.3 (dot-dashed), 0.5 (dashed), 0.7 (dotted) for intermediate blocking rule (i) illustrating the transition from concave downward to convex downward. (b) Final throughput, $V_{f}$ versus probability of partial coverage, $p_{p}$. In both cases we consider a feed of smaller particles than the pores, $a=0.9$, probability of adhesion, $p_{a 0}=0.1$, areal-dependent adhesion parameter, $k=0$, membrane thickness $h=10, Q^{*}=0$ so there are no caking effects, probability of arriving at a pore $p_{0}=1$, and a fractional partial reduction in flux, $\phi=0.25$.

the the affinity of the particles with the membrane surface when attempting to maximize the throughput. Such insight indicates how our simulations may be helpful in guiding the choice of membrane and operating regimes to optimize the filtration efficiency.

\section{Conclusions}

We have presented for the first time a discrete network model that is able to capture the inherent inter-relation among the various mechanisms via which a membrane may foul. In particular, the model captures pore constriction via the entry of particles into the pores and adhesion onto the pore walls, which allows entry of a finite number of particles before the pore becomes completely blocked. Following this, a layer of particles will be deposited on the surface that contribute further to membrane fouling. Each individual fouling mechanism possesses a characteristic signature when viewed as a graph of flux, $Q$, versus volumetric throughput, $V$. Specifically, internal membrane fouling or standard blocking was shown to yield a concavedownward graph, while complete blocking of pores by particles that cover pore entrances revealed itself through a linear $Q V$ signature. Finally, the deposition of a layer of particles on the membrane surface, or caking, induces a convex-downward curve representing the slow decay to zero flux. Conventional models that do not cater for the inter-relation between these fouling mechanisms typically yield convex-downward graphs, which capture a variety of experimental results well. However, for experiments in which these fouling mechanisms occur simultaneously we showed that this leads to a concave-downward $Q V$ signature and it is vital to accommodate the inter-relation (b)
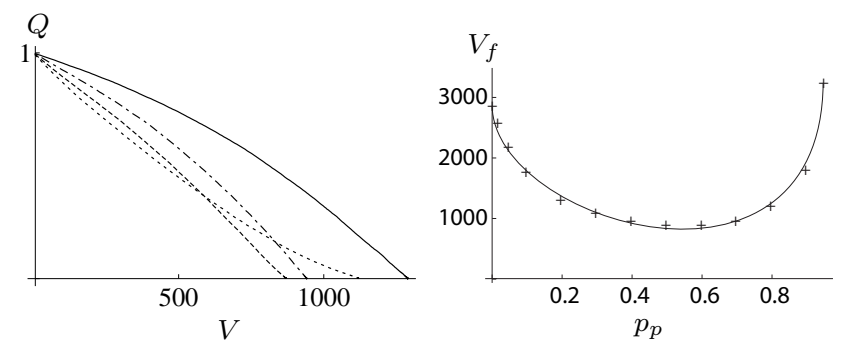

Figure 14: (a) Flux, $Q$, versus throughput, $V$, as the probability of landing partially on a pore, $p_{p}=0.2$ (solid), 0.4 (dot-dashed), 0.6 (dashed), 0.8 (dotted) for intermediate blocking rule (ii) illustrating the transition from concave downward to convex downward. (b) Final throughput, $V_{f}$ versus probability of partial coverage, $p_{p}$. In both cases we consider a feed of smaller particles than the pores, $a=0.9$, probability of adhesion, $p_{a 0}=0.1$, areal-dependent adhesion parameter, $k=0$, membrane thickness $h=10, Q^{*}=0$ so there are no caking effects, probability of arriving at a pore $p_{0}=1$, and a fractional partial reduction in flux, $\phi=0.25$.

among these fouling routes to capture the correct experimentally observed trends.

We analysed the parametric dependence of the $Q V$ signature on the key system parameters, namely internal pore adhesion, particle size, membrane thickness, probability of landing on the membrane and probability of partially obstructing a pore. We showed how the $Q V$ signature can vary significantly depending on these parameters but that many results may be mapped onto a master curve that allows us to make predictions about the microscopic physics from macroscopic measurements. In addition, we showed how the $Q V$ signature may be used to infer which stage of blocking a system is in at any given time.

The inclusion of intermediate pore blocking into the model introduced further new features to the system behaviour. In particular, as the likelihood of partially obstructing pore was varied we found that the $Q V$ signature was able to transition from the concave-downward shape that is characteristic of multiple fouling mechanisms to a more familiar convex downward structure.

The results of this model provide new insight on the microscopic blocking mechanisms that take place during membrane fouling, new methods for analysing macroscopic observations, and motivates further experiments. In particular, the modelling framework laid out here provides a foundation on which to explore more complex fouling phenomena, such as the inhomogeneities induced in the flow field due to the non-uniform build-up in the cake layer, as well as more complex fouling mechanisms arising for different particle types. The results should lead to new strategies for optimal filtration via appropriate membrane selection and operating regimes, as well as offering guidance for new filter design. 
Acknowledgements

The authors gratefully acknowledge helpful discussions from the Oxford-Pall 2013 In-House Study Group participants.

\section{References}

[1] C. Y. Tang, T. Chong, A. G. Fane, Colloidal interactions and fouling of NF and RO membranes: A review, Adv. Colloid and Interface Sci. 164 (1) (2011) 126-143.

[2] B. Ruth, Studies in filtration III. Derivation of general filtration equations, Ind. Eng. Chem. 27 (6) (1935) 708-723.

[3] J. C. Chen, Q. Li, M. Elimelech, In situ monitoring techniques for concentration polarization and fouling phenomena in membrane filtration, Adv. Colloid and Interface Sci. 107 (2) (2004) 83-108.

[4] H. Li, A. Fane, H. Coster, S. Vigneswaran, Direct observation of particle deposition on the membrane surface during crossflow microfiltration, J. Membrane Sci. 149 (1) (1998) 83-97.

[5] J. Altmann, S. Ripperger, Particle deposition and layer formation at the crossflow microfiltration, J. Membrane Sci. 124 (1) (1997) 119-128.

[6] M. Hamachi, M. Mietton-Peuchot, Experimental investigations of cake characteristics in crossflow microfiltration, Chem. Eng. Sci 54 (18) (1999) 4023-4030.

[7] A. P. Mairal, A. R. Greenberg, W. B. Krantz, L. J. Bond, Realtime measurement of inorganic fouling of RO desalination membranes using ultrasonic time-domain reflectometry, J. Membrane Sci. 159 (1) (1999) 185-196.

[8] L.-H. Cheng, Y.-C. Yang, J. Chen, Y.-H. Lin, S.-H. Wang, A new view of membrane fouling with 3D ultrasonic imaging techniques: Taking the canola oil with phospholipids for example, J. Membrane Sci. 372 (1) (2011) 134-144.

[9] T. Chilcott, M. Chan, L. Gaedt, T. Nantawisarakul, A. Fane, H. Coster, Electrical impedance spectroscopy characterisation of conducting membranes: I. Theory, J. Membrane Sci. 195 (2) (2002) 153-167.

[10] L. Gaedt, T. Chilcott, M. Chan, T. Nantawisarakul, A. Fane, H. Coster, Electrical impedance spectroscopy characterisation of conducting membranes: II. Experimental, J. Membrane Sci. 195 (2) (2002) 169-180.

[11] C. M. Bondy, C. Santeufemio, Analysis of fouling within microporous membranes in biopharmaceutical applications using latex microsphere suspensions, J. Membrane Sci. 349 (1) (2010) $12-24$.

[12] M. Ferrando, A. Rŏżek, M. Zator, F. Lopez, C. Güell, An approach to membrane fouling characterization by confocal scanning laser microscopy, J. Membrane Sci. 250 (1) (2005) 283-293.

[13] R. Chan, V. Chen, Characterization of protein fouling on membranes: opportunities and challenges, J. Membrane Sci. 242 (1) (2004) 169-188.

[14] M. Rahimi, S. Madaeni, M. Abolhasani, A. A. Alsairafi, CFD and experimental studies of fouling of a microfiltration membrane, Chem. Eng. and Processing: Process Intensification 48 (9) (2009) 1405-1413.

[15] A. Fouladitajar, F. Zokaee Ashtiani, A. Okhovat, B. Dabir, Membrane fouling in microfiltration of oil-in-water emulsions; a comparison between constant pressure blocking laws and genetic programming (GP) model, Desal. 329 (2013) 41-49.

[16] Q.-F. Liu, S.-H. Kim, Evaluation of membrane fouling models based on bench-scale experiments: a comparison between constant flowrate blocking laws and artificial neural network (ANNs) model, J. Membrane Sci. 310 (1) (2008) 393-401.

[17] M. Wessling, Two-dimensional stochastic modeling of membrane fouling, Separation and Purification Technol. 24 (3) (2001) 375-387.

[18] G. De Luca, F. Bisignano, F. Paone, S. Curcio, Multi-scale modeling of protein fouling in ultrafiltration process, J. Membrane Sci. 452 (2014) 400-414.

[19] A. Grenier, M. Meireles, P. Aimar, P. Carvin, Analysing flux decline in dead-end filtration, Chem. Eng. Research and Design 86 (11) (2008) 1281-1293.

20] W. Bowen, J. Calvo, A. Hernandez, Steps of membrane blocking in flux decline during protein microfiltration, J. Membrane Sci. 101 (1) (1995) 153-165.

[21] P. Hermans, H. Bredee, Zur kenntnis der filtrationsgesetze, Recueil des Travaux Chimiques des Pays-Bas 54 (9) (1935) 680700 .

[22] A. Koltuniewicz, R. Field, T. Arnot, Cross-flow and deadend microfiltration of oily-water emulsion. Part I: Experimental study and analysis of flux decline, J. Membrane Sci. 102 (1995) 193-207.

[23] E. M. Tracey, R. H. Davis, Protein fouling of track-etched polycarbonate microfiltration membranes, J. Colloid and Interface Sci. 167 (1) (1994) 104-116.

[24] W. Yuan, A. Kocic, A. L. Zydney, Analysis of humic acid fouling during microfiltration using a pore blockage-cake filtration model, J. Membrane Sci. 198 (1) (2002) 51-62.

[25] F. Zokaee, T. Kaghazchi, A. Zare, Cell harvesting by microfiltration in a deadend system, Process Biochem. 34 (8) (1999) 803-810.

[26] E. Iritani, Y. Mukai, Y. Tanaka, T. Murase, Flux decline behavior in dead-end microfiltration of protein solutions, J. Membrane Sci. 103 (1) (1995) 181-191.

[27] C.-C. Ho, A. L. Zydney, A combined pore blockage and cake filtration model for protein fouling during microfiltration, J. Colloid and Interface Sci. 232 (2) (2000) 389-399.

[28] L. Palacio, C.-C. Ho, A. L. Zydney, Application of a poreblockagecake-filtration model to protein fouling during microfiltration, Biotechnol. Bioeng. 79 (3) (2002) 260-270.

[29] G. Bolton, D. LaCasse, R. Kuriyel, Combined models of membrane fouling: Development and application to microfiltration and ultrafiltration of biological fluids, J. Membrane Sci. 277 (1) (2006) 75-84.

[30] C. Duclos-Orsello, W. Li, C.-C. Ho, A three mechanism model to describe fouling of microfiltration membranes, J. Membrane Sci. 280 (1) (2006) 856-866.

[31] G. R. Bolton, A. W. Boesch, M. J. Lazzara, The effects of flow rate on membrane capacity: Development and application of adsorptive membrane fouling models, J. Membrane Sci. 279 (1) (2006) 625-634.

[32] S. Giglia, G. Straeffer, Combined mechanism fouling model and method for optimization of series microfiltration performance, J. Membrane Sci. 417 (2012) 144-153.

[33] Y. S. Polyakov, Depth filtration approach to the theory of standard blocking: Prediction of membrane permeation rate and selectivity, J. Membrane Sci. 322 (1) (2008) 81-90.

[34] P. Apel, Track etching technique in membrane technology, Radiation Measurements 34 (1) (2001) 559-566.

[35] H. Ockendon, J. R. Ockendon, Viscous flow, Vol. 13, Cambridge University Press, 1995 